\title{
On the Dimensions of Certain Incommensurably Constructed Sets
}

\author{
J. J. P. Veerman and B. D. Stošić
}

\section{CONTENTS}

1. Introduction

2. Methods for Commensurably Constructed Sets

3. Methods for Incommensurably Constructed Sets

4. Numerical Results

Acknowledgements

References
It is known that the Hausdorff dimension of the invariant set $\Lambda_{\mathrm{t}}$ of an iterated function system $\mathcal{F}_{t}$ on $\mathbb{R}^{n}$ depending smoothly on a parameter $t$ varies lower-semicontinuously, but not necessarily continuously. For a specific family of systems we investigate numerically the conjecture that discontinuities in the dimension only arise when in some iterate of the iterated function system two or more branches coincide. This happens in a dense set of codimension one. All other points are conjectured to be points of continuity.

\section{INTRODUCTION}

Let $\mathcal{F}=\left\{f_{i}\right\}_{i=0}^{N}$ be a collection of smooth contracting diffeomorphisms of $\mathbb{R}^{n}$. Such a collection is often called an iterated function system, since we are interested in its properties under iteration. There exists a unique compact invariant set $\Lambda$ with the property

$$
\Lambda=\bigcup_{i} f_{i}(\Lambda)
$$

and this set supports a natural invariant probability measure $\nu_{t}$ (see Section 2 for the meaning of natural). For all these statements, see [Hutchinson 1981], for example.

We now consider a family $\left\{\mathcal{F}_{t}\right\}$ of iterated functions systems, depending smoothly on a real parameter $t$, together with the associated invariant sets $\Lambda_{t}$ and probability measures $\nu_{t}$. Under certain weak conformality conditions for systems in dimension greater than 1, the Hausdorff dimension and the Lebesgue measure of $\Lambda_{t}$ vary semicontinuously [Veerman and Jonker 1997]. That this is the best possible general result is illustrated by the family of systems discussed in this paper (see Theorem 1.1). The question that arises is: where and how often do the discontinuities arise? Can we say that in some 
sense the dimension and the measure of the set are typically continuous?

The example family we will discuss in this paper is $\mathcal{F}_{t}$, for $t \in[0,1 / 2]$, where $\mathcal{F}=\left\{f_{i}\right\}_{i=0}^{2}$ is given by

$$
\begin{aligned}
& f_{0}(x)=\frac{1}{3} x, \\
& f_{1}(x)=\frac{1}{3}(x+t), \\
& f_{2}(x)=\frac{1}{3}(x+1) .
\end{aligned}
$$

The Hausdorff dimension Hdim and the Lebesgue measure $\mu$ of the invariant set depend on $t$. The following striking result was stated in [Veerman and Jonker 1997], although the most important parts of it were proved in older papers (referenced there). A proof can also be found in [Kenyon 1997].

Theorem 1.1. Let $\mathcal{F}_{t}$ be the system just described. Then:

(i) If $t=p / q$ is rational and $p q=2 \bmod 3$ then $\mu(t)=1 / q$.

(ii) If $t=p / q$ is rational and $p q \neq 2 \bmod 3$ then $\operatorname{Hdim}(t)<1$.

(iii) For all irrational $t, \mu(t)=0$.

(iv) For almost all $t, \operatorname{Hdim}(t)=1$.

We shall see in Section 2 that whenever the system respects an (invertible) affine image $L$ of $\mathbb{Z}^{n}$ in the sense that $\bigcup_{i} f_{i}^{-1}(L) \subseteq L$ (we call this commensurably constructed), then efficient algorithms to calculate, or estimate, the dimension are available. For the family under consideration, it is easy to see that $\Lambda_{t}$ is commensurably constructed if and only if $t$ is rational. The following conjecture (attributed to Furstenberg in [Kenyon 1997]) stipulates that discontinuities should only occur at rational values of $t$.

Conjecture 1.2. For all irrational $t, \operatorname{Hdim}(t)=1$.

(Note that Theorem 1.1 already guarantees the continuity of the measure at irrational values of $t$.)

This article provides numerical and heuristic evidence that for irrational values of $t$ (in particular the golden mean) the dimension of the invariant set equals 1 . Thus in Section 2 we outline our algorithms used to do the numerics. In Section 3 we prove that for certain Liouville numbers $t$, the invariant set indeed has dimension 1 . The main purpose of this paper is to present evidence in support of Conjecture 1.2. This is done in Section 4.

\section{METHODS FOR COMMENSURABLY CONSTRUCTED SETS}

This section lists some methods to calculate or estimate the dimension for rational values of $t$. As proved in [Veerman and Jonker 1997] (see also Section 3), the Hausdorff dimension of the invariant set (for the sets under consideration) is equal to its limit capacity. The algorithms we discuss will thus only have to calculate the limit capacity (or box dimension).

Suppose $t$ is rational, say $p / q$. Then by the affine coordinate transform $x \rightarrow x / q$ we may map the iterated function system to the following:

$$
\begin{aligned}
& f_{0}(x)=\frac{1}{3} x, \\
& f_{1}(x)=\frac{1}{3}(x+p), \\
& f_{2}(x)=\frac{1}{3}(x+q) .
\end{aligned}
$$

An equivalent definition of the invariant set of this system is the set of points

$\Lambda(3,\{0, p, q\})=\left\{x \in \mathbb{R}: x=\sum_{i=1}^{\infty} 3^{-i} r_{i}, r_{i} \in\{0, p, q\}\right\}$.

We can thus approximate the set by considering the points that can be written as $\sum_{i=0}^{k-1} 3^{-i} r_{i}, r_{i} \in$ $\{0, p, q\} \equiv R$. A simpler way of stating this is

$$
\Lambda_{k}=3^{-k} \sum_{i=0}^{k-1} 3^{i} R \text { and } \Lambda=\lim \Lambda_{k},
$$

where the summation here is a sum of sets. The sum of two sets $A$ and $B$ is defined as follows:

$$
Z=A+B \stackrel{\text { def }}{=}\{z: z=a+b, a \in A, b \in B\} .
$$

(See [Hacon et al. 1994; Veerman 1995; 1998] for details.) The box dimension is calculated by counting the number of distinct integers in $\left\{\sum_{i=0}^{k-1} 3^{i} R\right\}$. Let $\gamma_{k}$ denote this number.

Proposition 2.1. The Hausdorff dimension of the invariant set of the system $(3,\{0, p, q\})$ is

$$
\lim _{k \rightarrow \infty} \frac{\log \gamma_{k}}{\log 3^{k}}
$$

The method of calculating the dimension in which we are interested here is a refinement of this. It was developed independently (and slightly earlier) by Rao and Wen [1998] and proved by different methods. 
Note that if $R$ consists of integers, the expressions in $\left\{\sum_{i=0}^{k-1} 3^{i} R\right\}$ have integer values. In these cases we may define the maps $T_{k}$ and $t_{k}$ from the integers to the nonnegative integers:

$$
\begin{aligned}
& T_{k}(l)=\#\left\{\sum_{i=0}^{k-1} 3^{i} R=l\right\}, \\
& t_{k}(l)=\min \left\{T_{k}(l), 1\right\} .
\end{aligned}
$$

By a word $w\left(\right.$ in $\left.t_{k}\right)$ we mean an ordered set of consecutive values of $t_{k}$,

$$
\left\{t_{k}\left(i_{0}\right), t_{k}\left(i_{0}+1\right), \ldots, t_{k}\left(i_{0}+|w|-1\right)\right\},
$$

where $|w|$ is the length of the word, and such that $i_{0}, i_{0}+1, \ldots, i_{0}+|w|-1$ is maximal with respect to the condition that less than $q / 2-1$ consecutive values are zero. Similarly we can define words in $T_{k}$.

The sentence $t_{k+1}$ is created from the sentence $t_{k}$ by multiplying by 3 and adding $R$. If we apply this operation to a word $w$ in $t_{k}$ we refer to the resulting part of $t_{k+1}$ as the offspring of $w$. It is easy to see that if $w_{1}$ and $w_{2}$ are consecutive words in $t_{k}$ then $3 w_{1}+q$ and $3 w_{2}$ are separated by $q / 2-1$ or more zeros. Thus the offspring of a word must consist of words.

If we let $\left\{w_{i}\right\}$ be a list of all the words occurring in $\bigcup_{k} t_{k}$, then we can write the offspring of $w_{i}$ as a (finite) linear combination $\sum_{i} d_{i j} w_{j}$. Let $\lambda_{D}$ be the leading eigenvalue of the matrix $D=\left(\left(d_{i j}\right)\right)$.

Theorem 2.2. If the number of words in the development of the system $(3,\{0, p, q\})$ is finite, then the invariant set of that system has Hausdorff dimension equal to $\log \lambda_{D} / \log 3$.

Remark. The numerical algorithm we used to calculate $\lambda_{D}$ does not terminate unless the number of distinct words in $\bigcup_{k} T_{k}$ is finite and smaller than a realistic limit set by memory limitations: if this is not the case, the algorithm does not finish its calculation of the values of the entries of the transition matrix $D$. In fact, in [Rao and Wen 1998] it is proved that if the dimension of the invariant set of the system $(3,\{0, p, q\})$ is smaller than 1 , then the number of words is always finite. We do not include this proof here, since in practice the calculational limits are quickly exhausted (see Section 4).

Proof of the theorem. Supposing the number of distinct words is finite, we obtain a finite matrix $D$.
Moreover, the matrix is primitive, since all words were constructed from the initial word $\{0\}$. By the Perron-Frobenius theorem, there is a unique leading eigenvalue $\lambda_{D}>0$. The associated eigenvector $v$ gives the asymptotic distribution of words. For large $k$, the number of occurrences of every type of word is multiplied by $\lambda_{D}$ from one level to the next. But that means that the number $\gamma_{k}$ is multiplied by $\lambda_{D}$. Now apply Proposition 2.1.

The new method thus consists in identifying words and expressing its offspring in terms of the original words and calculating the eigenvalue of the corresponding transition matrix. If

$$
w=\left\{t_{k}\left(i_{0}\right), t_{k}\left(i_{0}+1\right), \ldots, t_{k}\left(i_{0}+|w|-1\right)\right\}
$$

is a word, we can specify this word by listing, in order, the distances of the non-zero entries from the first entry. For example, the word $\{1,1,0,1,0,1\}$ for $(3,\{0,1,5\})$ would be denoted as $\{0,1,3,5\}$. Using this notation, we see that for the system $(3,\{0,1,3\})$, where no consecutive zeroes are allowed, the development yields only 2 distinct words, namely $\{0,1\}$ and $\{0\}$. This line of reasoning leads to the following.

$$
\begin{aligned}
\{0\} & \rightarrow\{0,1,3\} \equiv\{0\} \cup\{0,1\}, \\
\{0,1\} & \rightarrow\{0,1,3,4,6\} \equiv\{0\} \cup 2\{0,1\} .
\end{aligned}
$$

We can write down the transition matrix $D$ as

$$
D=\left(\begin{array}{ll}
1 & 1 \\
1 & 2
\end{array}\right)
$$

We conclude that the dimension of this set equals

$$
\operatorname{Hdim}\left(\Lambda_{1 / 3}\right)=\log ((3+\sqrt{5}) / 2) / \log 3 \approx 0.867 \ldots,
$$

since $(3+\sqrt{5}) / 2$ is the leading eigenvalue of the matrix $D$. The same result was obtained earlier in [Keane and Smorodinski 1997].

As we noted earlier, and can be checked in Section 4, the algorithm above rather quickly exhausts our computational limits. For our purposes, it is sufficient to have a lower bound estimate of the Hausdorff dimension. This can be done much more efficiently as we shall now see.

We may follow [Hutchinson 1981] and adopt the view that the system $\mathcal{F}_{t}$, as given in $(1-1)$, acts lin- 
early on the space of probability measures on the interval as follows ( $V$ is an interval):

$$
\mathcal{F}_{t} \mu(V)=\frac{1}{3} \sum_{n=0}^{2} \mu\left(f_{n}^{-1}(V)\right),
$$

where the $f_{n}$ are given in equation (1-1). If we put an appropriate metric on the space of measures, $\mathcal{F}_{t}$ is a (uniform) contraction (see [Hutchinson 1981]).

Define a sequence $\nu_{p / q}^{(k)}$ of probability measures associated with the system $(3,\{0, p / q, 1\})$.

$$
\nu_{p / q}^{(k)}(V)=3^{-k} \sum_{q^{-1} 3^{-k} i \in V} T_{k}(i),
$$

where $T_{k}$ is the function defined before for the system $(3,\{0, p, q\})$ (note the difference in the systems).

The next result can be found in [Hutchinson 1981], for example.

Lemma 2.3. The limit $\lim _{k \rightarrow \infty} \nu_{p / q}^{(k)}$ is the unique fixed point of $\mathcal{F}_{p / q}$.

Proof. It is sufficient to prove that $\mathcal{F}_{p / q} \nu_{p / q}^{(k)}=\nu_{p / q}^{(k+1)}$. Write $R=\{0, p, q\}$ and observe that it consists of integers. From the definition of $T$, we see that

$$
\begin{aligned}
T_{k+1}(l) & =\#\left\{\sum_{i=0}^{k-1} 3^{i} R+3^{k} R=l\right\} \\
& =\sum_{j} T_{k}(j) \#\left\{j+3^{k} R=l\right\} .
\end{aligned}
$$

Combining this with (2-4) we obtain

$$
\begin{aligned}
& \nu^{(k+1)}(V)=3^{-k-1} \sum_{q^{-1} 3^{-k-1}} T_{k+V}(i) \\
& =3^{-k-1} \sum_{q^{-1} 3^{-k-1}} \sum_{i \in V} T_{k}(j) \#\left\{j+3^{k} R=i\right\} .
\end{aligned}
$$

We may rewrite this as

$$
\nu^{(k+1)}(V)=\frac{1}{3} 3^{-k} \sum_{j \in Q} T_{k}(j),
$$

where the summation set $Q$ consists of those $j$ such that $q^{-1} 3^{-k-1}\left(j+3^{k} R\right)=q^{-1} 3^{-k} i \in V$ implies

$$
q^{-1} 3^{-k} j \in 3 V-R / q .
$$

Thus by (1-1) we see that

$$
\nu^{(k+1)}(V)=\frac{1}{3} \sum_{n} \nu^{(k)}\left(f_{n}^{-1}(V)\right),
$$

in accordance with $(2-3)$. Together with the fact that $\mathcal{F}$ is a contraction this proves the result.
Returning to the invariant set of $(2-1)$, define

$$
S \stackrel{\text { def }}{=}\{\Lambda-\Lambda\} \cap \mathbb{Z}
$$

that $S$ is the set of integer differences contained in $\Lambda$. Writing $\Lambda-\Lambda$ as a set of numbers on the base 3 , as in $(2-2)$, we easily see that $\Lambda-\Lambda$ is the invariant set of the system $(3,\{0, \pm p, \pm(q-p), \pm q\})$. An easy argument shows that this is the same as the invariant set of $(3,\{0, \pm q\})$, which is given by $[-q / 2, q / 2]$.

Let $\mathbb{R}^{S}$ be the vector space obtained by associating a basis vector $e_{l}$ to each element $l$ of $S$. Following [Veerman 1998], define a linear map $\mathcal{T}: \mathbb{R}^{S} \rightarrow \mathbb{R}^{S}$, the transition operator for the differences, whose matrix elements are given by:

$$
\mathcal{T}_{i j} \stackrel{\text { def }}{=} \sum_{l} T_{1}(l) T_{1}(l+i-3 j),
$$

where $i$ and $j$ are in $S$. This operator plays a fundamental role in the theory of iterated function systems, although it goes by very different names and formulations in different works such as [Pollicott and Simon 1995; Lagarias and Wang 1996; Veerman 1996; 1998; Kenyon 1997; Solomyak 1995]. In words, it is the matrix whose $(i, j)$-th entry corresponds to the number of differences in $R$ that are equal to $i-3 j$. We will call this operator the difference operator. One may reduce the dimension of the matrix by a factor of almost 2 , by exploiting the fact that we are interested only in how $\mathcal{T}$ operates on even vectors, $v_{-i}=v_{i}$. This is because for every difference $r_{1}-r_{2}=d$, we must have a difference $-d$ in the sequence; see [Veerman 1998]. Define the reduced difference operator $\mathcal{T}^{(R)}$ by setting, for $i \geq 0$,

$$
\mathcal{T}_{i, j}^{(R)}= \begin{cases}\mathcal{T}_{i, j}+\mathcal{T}_{i,-j} & \text { if } j>0, \\ \mathcal{T}_{i, j}^{(R)}=\mathcal{T}_{i, j} & \text { if } j=0 .\end{cases}
$$

Then $\mathcal{T}^{(R)}$ acts on the reduced space as the original $\mathcal{T}$ does on symmetric vectors. From now on we use the abbreviation $\mathcal{T}$ for this operator as well.

The content of the next lemma is that the number of differences in $\left\{\sum_{i=0}^{k-1} 3^{i} R\right\}$ equal to $d$ may be calculated by iterating the matrix $\mathcal{T}$. This is very similar to what is proved in [Veerman 1998, Section 3] in greater generality. We give a simplified proof for completeness. Recall that $e_{0}$ is the standard basis vector in $\mathbb{R}^{S}$ associated with $0 \in S$. 
Lemma 2.4. The growth rate of $\sum_{i} T_{k}(i)^{2}($ as $k \rightarrow \infty)$ is equal to the growth rate $\kappa$ of $\left|\mathcal{T}^{k} e_{0}\right|$.

Proof. Start by observing that

$$
\begin{aligned}
T_{k+1}(i) & =\#\left\{R+3 \sum_{l=0}^{k-1} 3^{l} R=i\right\} \\
& =\sum_{j} T_{1}(i-3 j) T_{k}(j) .
\end{aligned}
$$

Using this formula, we see that for each $d \in S$

$$
\begin{aligned}
& \sum_{i} T_{k+1}(i) T_{k+1}(i+d) \\
& =\sum_{i} \sum_{j, n} T_{1}(i-3 j) T_{1}(d+i-3 n) T_{k}(j) T_{k}(n),
\end{aligned}
$$

where all the summations are over $\mathbb{Z}$. Eliminate $i$ and $j$ in favor of $p$ and $r$ by setting:

$$
i=p+3 n-3 r, \quad j=n-r,
$$

obtaining

$$
\begin{aligned}
& \sum_{i \in \mathbb{Z}} T_{k+1}(i) T_{k+1}(i+d) \\
& =\sum_{n, p, r \in \mathbb{Z}} T_{1}(p) T_{1}(p+d-3 r) T_{k}(n) T_{k}(n-r) \\
& =\sum_{r \in \mathbb{Z}}\left(\sum_{p \in \mathbb{Z}} T_{1}(p) T_{1}(p+d-3 r)\right)\left(\sum_{n \in \mathbb{Z}} T_{k}(n) T_{k}(n-r)\right) .
\end{aligned}
$$

By symmetry, we may change $r$ to $-r$ in the rightmost sum. Also, $T_{1}(p) T_{1}(p+d-3 r)$ is non-zero only if $s-M r \in R-R$. Since $d-3 r$ must be in $R-R \subset[-q, q]$ and $d \in S$, it is easy to see that $r$ must be in $[-q / 2, q / 2]$. Thus by using $(2-5)$ we see that

$$
\begin{aligned}
\sum_{i} T_{k+1}(i) T_{k+1}(i+d) & \\
& =\sum_{r \in S} \mathcal{T}_{d, r}\left(\sum_{n \in \mathbb{Z}} T_{k}(n) T_{k}(n+r)\right) .
\end{aligned}
$$

By a recursion argument we get

$$
\sum_{i} T_{k}(i) T_{k}(i+d)=\left(\mathcal{T}^{k} e_{0}\right)_{d \text {-th component }} .
$$

To derive the lemma it is sufficient to notice that the growth rate of this expression is independent of $d$, because it is an eigenvalue of $\mathcal{T}$. Thus we may set $d=0$.

We note that one can easily prove that the eigenvalue of $\mathcal{T}$ referred to in this proof is the leading one.
However, this fact will not be used in the numerical part of this work. In fact, the algorithm we will be using (see equation (4-1)) is based on equation $(2-6)$.

The next result gives an efficient way to estimate the dimension of the sets under consideration. It is apparently new, although a related one has appeared in [Lau 1993], where a very different method of proof is used.

Theorem 2.5. Denote by $\kappa$ the leading eigenvalue of the difference operator associated with the system $(3,\{0, p, q\})$. Then

$$
\operatorname{Hdim}(\Lambda(3,\{0, p, q\})) \geq \frac{\log (9 / \kappa)}{\log 3} .
$$

Proof. Note that $\nu_{p / q}^{(n)}(\Lambda)=1$ for all $n$. Denote by $x^{(n)}(\Lambda)$ and $v^{(n)}(\Lambda)$ the restrictions of $T_{n}$ and $t_{n}$ to the $i$-th components where $q^{-1} 3^{-n} i \in \Lambda$. Now Hölder's inequality gives us the estimate

$$
\cos \theta_{n} \stackrel{\text { def }}{=} \frac{\sum v_{i} x_{i}}{\sqrt{\sum v_{i}^{2}} \sqrt{\sum x_{i}^{2}}}=\frac{\sum x_{i}}{\sqrt{\sum v_{i}} \sqrt{\sum x_{i}^{2}}} \leq 1,
$$

where we have dropped the superscripts $(n)$ and where, of course, the summations are over the indices $i$ such that $q^{-1} 3^{-n} i \in \Lambda$. From the definitions above we know that $3^{-n} \sum x_{i}$ estimates $\nu^{(n)}(\Lambda)$. To take the limit as $n \rightarrow \infty$ recall that $\nu^{(n)}$ converges to $\nu$ by Lemma 2.3. Also note that $\sum v_{i}$ estimates the number $N_{n}$ of intervals of size $q^{-1} 3^{-n}$ needed to cover $\Lambda$. According to the previous lemma, the growth rate of $\sum x_{i}^{2}$ is given by $\kappa$, the leading eigenvalue of $\mathcal{T}$.

$$
\begin{aligned}
\lim _{n \rightarrow \infty} \frac{1}{n} \log \cos \theta_{n} & =\lim _{n \rightarrow \infty} \frac{1}{n} \log \left(\frac{3^{n} \nu_{p / q}^{(n)}(\Lambda)}{\sqrt{N_{n}} \sqrt{\kappa^{n}}}\right) \\
& =\frac{\log 3}{2}\left(\frac{\log 9 / \kappa}{\log 3}-\operatorname{box} \operatorname{dim}(\Lambda)\right) \\
& \leq 0
\end{aligned}
$$

This gives an estimate for the box dimension of $\Lambda$ :

$$
\text { box } \operatorname{dim}(\Lambda) \geq \frac{\log (9 / \kappa)}{\log 3} .
$$

Since $\Lambda$ is contained in $\Lambda$ it also is an estimate for the box dimension of $\Lambda$. But the box dimension of $\Lambda$ equals its Hausdorff dimension by [Veerman and Jonker 1997]. 
By way of illustration, we work out one example. The reduced difference operator associated with the system $(3,\{0,1,3\})$ is easily calculated, noting that $S=\{0,1\}$ (for the reduced difference operator):

$$
\mathcal{T}=\left(\begin{array}{ll}
3 & 2 \\
1 & 1
\end{array}\right)
$$

so

$$
\mathcal{T}\left(\begin{array}{l}
1 \\
0
\end{array}\right)=\left(\begin{array}{l}
3 \\
1
\end{array}\right) \quad \text { and } \quad \mathcal{T}^{2}\left(\begin{array}{l}
1 \\
0
\end{array}\right)=\left(\begin{array}{c}
11 \\
4
\end{array}\right) .
$$

Since in this case we have $\kappa=2+\sqrt{3}$, the lower estimate is

$$
d \geq \frac{\log (9 /(2+\sqrt{3}))}{\log 3}=0.801253262 .
$$

This is less than the exact value of the dimension of this invariant set found before.

When the invariant set has positive measure (see Theorem 1.1), its dimension is trivially equal to 1 . In this case we may calculate the dimension of the boundary of the invariant set (this idea was first developed in [Veerman 1998] in a more general context). An eigenvalue of $\mathcal{T}$ is called special if it is real and contained in $[1,3)$.

Theorem 2.6. Let $(3,\{0, p, q\})$ be such that $p q=2$ $\bmod 3$. The associated matrix $\mathcal{T}$ always has at least one special eigenvalue. If we call the leading special eigenvalue $\lambda$, then the Hausdorff dimension of $\partial \Lambda$ (the boundary of $\Lambda$ ) is given by

$$
\operatorname{Hdim}(\partial \Lambda)=\frac{\log \lambda}{\log 3} .
$$

Since the system $(3,\{0,1,3\})$ is not of the form required by the theorem, we consider instead the system $(3,\{0,8,13\})$. With a little work one can see that the reduced difference operator becomes:

$$
\mathcal{T}=\left(\begin{array}{lllllll}
3 & 0 & 0 & 0 & 0 & 0 & 0 \\
0 & 0 & 1 & 1 & 1 & 0 & 0 \\
0 & 1 & 1 & 0 & 0 & 1 & 0 \\
0 & 3 & 0 & 0 & 0 & 0 & 0 \\
0 & 0 & 0 & 2 & 1 & 0 & 0 \\
1 & 1 & 0 & 0 & 0 & 0 & 1 \\
0 & 0 & 3 & 0 & 0 & 0 & 0
\end{array}\right)
$$

To obtain the next-to-leading eigenvalue, one may set the $\mathcal{T}_{00}=0$ as in [Veerman 1998] and use the same algorithm as in equation (4-1).

\section{METHODS FOR INCOMMENSURABLY CONSTRUCTED SETS}

We present an estimate for the dimension for $t$ in a certain class of irrationals. This estimate is a simple consequence of a general result which we give first.

Theorem 3.1 [Veerman and Jonker 1997]. (i) For the set $\Lambda_{t}$, the Hausdorff dimension equals the limit capacity (or box dimension).

(ii) The Hausdorff dimension is a lower semicontinuous function of $t$.

(iii) The Lebesgue measure of $\Lambda_{t}$ is an upper semicontinuous function of $t$.

(iv) For $d$ greater than the upper box dimension of $\partial \Lambda\left(\mathcal{F}_{t_{0}}\right)$, and for any $\varepsilon>0$ and $t$ sufficiently close to $t_{0}$, we have true:

$$
\mu(t) \leq \mu\left(t_{0}\right)+\varepsilon\left|t-t_{0}\right|^{1-d} .
$$

Let $\mathcal{O}_{\nu}$ be the subset of reals $t \in[0,1]$ such that

(i) $t$ is irrational and for some $C>0$ there is an infinite number of rationals satisfying $|t-p / q| \leq$ $C / q^{\nu}$, and

(ii) the equation above has an infinite number of solutions $p / q$ with $p q=2 \bmod 3$.

Theorem 3.2. When $t \in \mathcal{O}_{\nu}$, the Hausdorff dimension of $\Lambda_{t}$ is greater than or equal to $1-1 / \nu$.

Remark. In [Kenyon 1997] the same statement is proved differently.

Proof. Suppose that $t$ in $\mathcal{O}_{\nu}$, and let $d_{0}$ be the box dimension of $\Lambda_{t}$. By Theorem 3.1, $d_{0}=\operatorname{Hdim}(t)$. By the same theorem, and using the definition of $\mathcal{O}_{\nu}$, we conclude that for $d>d_{0}$

$$
\mu\left(\frac{p}{q}\right) \leq \mu(t)+\varepsilon\left|t-\frac{p}{q}\right|^{1-d}
$$

implies

$$
\frac{1}{q} \leq\left(\frac{C}{q^{\nu}}\right)^{1-d}
$$

Here we have also used Theorem 1.1 twice, namely $\mu(t)=0$, and $\mu\left(\frac{p}{q}\right)=1 / q$. The last equation implies the result.

Corollary 3.3. If $t \in \bigcap_{\nu} \mathcal{O}_{\nu}$ then $\operatorname{Hdim}(t)=1$.

The set $\bigcap_{\nu} \mathcal{O}_{\nu}$ is contained in the set of Liouville numbers, which though uncountable has Hausdorff dimension zero [Oxtoby 1980]. This is different from statement (iv) of Theorem 1.1, since now we identify 
a set of irrationals where the dimension is one. In the proof of the theorem it is important that the irrational number $t$ for which we obtain an estimate is close to certain rationals. In particular, the theorem does not apply to $t=(\sqrt{5}-1) / 2$, the golden mean.

\section{NUMERICAL RESULTS}

We now describe certain numerical computations, performed using the methods outlined in the previous sections, which support the conjecture that the Hausdorff dimension of the invariant set for the system $(3,\{0, t, 1\})$, where $t=g$ is the golden mean, equals 1 . We discuss each figure and summarize our results.

In these numerical calculations we employ a convenient algorithm to calculate the appropriate eigenvalue $\lambda$ of a matrix $D$ :

$$
\lambda=\lim _{n \rightarrow \infty} \frac{\left|D^{n+1} v\right|}{\left|D^{n} v\right|}
$$

where, of course, $v=(1,0, \ldots, 0)^{\dagger}$, represents the word $\{0\}$ (in the calculation according to Theorem 2.2 ) or the difference vector whose only difference is zero (in the calculation according to Theorem 2.5).

To create Figure $1,3^{18} \approx 4 \cdot 10^{8}$ points of the invariant set for the system $(3,\{0, g, 1\})$ were laid out on the interval. We then performed a standard box-counting procedure to estimate the dimension. As can be seen in Figure 1, the estimate on the dimension is not very accurate and even seems to converge to a number less than 1 .

Claim. The conventional box-counting procedure to estimate the dimension may occasionally lead to erroneous conclusions, even when applied to relatively simple subsets of the line (such as the one under consideration).

For lack of reliable computational methods applicable to the incommensurate case, we study the dimensions of a sequence of commensurably constructed sets converging to the desired set (with $t=g$ ). The results are displayed in Figure 2. First we calculated the exact dimension of the invariant sets associated to the systems $\left(3,\left\{0, f_{n-1} / f_{n}, 1\right\}\right)$ using the word counting algorithm of Section 2. Here $f_{n-1} / f_{n}$ is the $n$-th Farey approximant to the golden mean ( $f_{1}=1, f_{2}=2$ are the Fibonacci numbers).

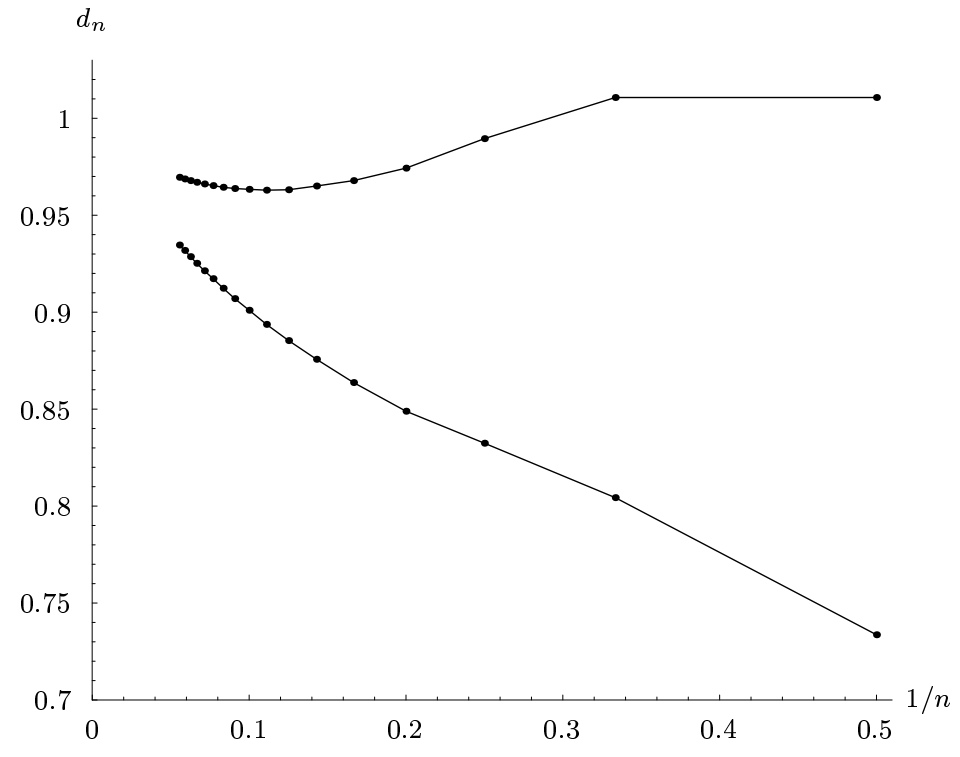

FIGURE 1. Direct calculation of the box dimension. Top curve: $\log \left(N_{n}\right) / \log \left(\left(3^{n}+1\right) / 2\right)$. Bottom curve: $\log \left(N_{n}\right) /(n \log 3)$.

The number of distinct words in its grammar increases dramatically and the algorithm ceases to be practicable beyond $n=10$. In fact, for $n=9$ the grammar consists of 8954 words with the maximum word length of 20794 letters (integers), the main restriction in continuing the sequence being the available computer memory for dictionary storage. Finding the corresponding point in Figure 2 therefore involves calculating the leading eigenvalue of a $8954 \times 8954$ transition matrix. To continue the sequence, we used the more efficient algorithm described in Theorem 2.5 giving the lower bound of the dimension. In this case, the computational difficulties arise from computational time rather then memory requirements, and here we have performed calculations up to $n=18$. Note that every fourth dimension is equal to 1 as it satisfies the criterion given in Theorem 1.1(i).

The apparent convergence of the sequence of dimensions in Figure 2 is of course no proof that the dimension is continuous at $t=g$. We set out to compare its behavior with that of at least two other sequences, namely one for which we know the dimension is continuous at its limit point $(t=1 / 2)$, and one for which we know that there is a discontinuity $(t=0)$.

At $t=1 / 2$ the dimension is continuous, by Theorem 1.1. Figure 3 displays the dimensions of the 


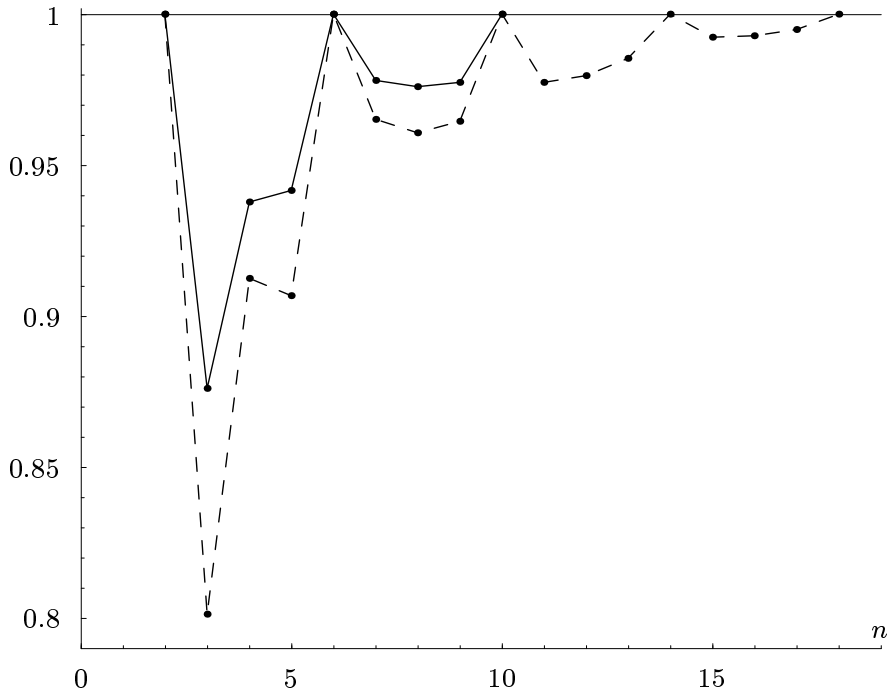

FIGURE 2. Dimension calculation when $t \rightarrow g$. The system is $\left\{3,0, f_{n-1}, f_{n}\right\}$; the solid curve gives the actual dimension, and the dashed curve the lower bound of the text.

invariant sets of the systems $(3,\{0, n /(2 n+1), 1\})$. Although only relatively few exact dimensions could be calculated, they do appear to converge to 1 . The sequence of lower bounds displayed in the same picture clearly converges to 1 . (In fact, we calculated the lower bounds for the dimensions up to $n=500$ and the convergence persists, the last value for the bound being 0.999507.)

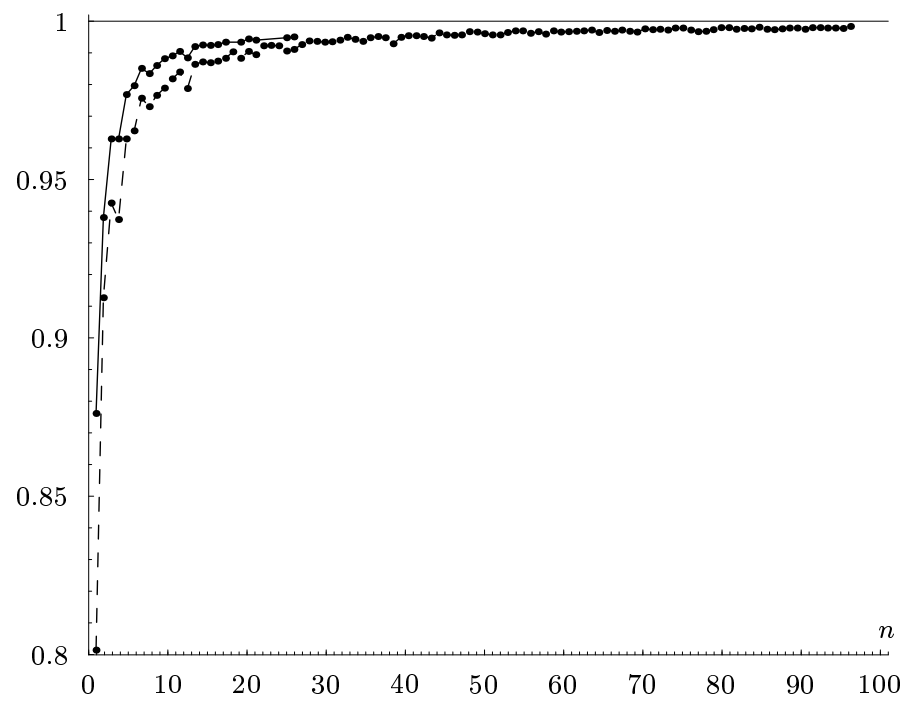

FIGURE 3. Dimension calculation when $t \rightarrow 1 / 2$. The system is $(3,\{0, n /(2 n+1), 1\})$; the upper (solid) curve gives the actual dimension, and the dashed curve that extends to the right gives the lower bound.
At $t=0$ the dimension is discontinuous. Figure 4 plots both the exact values and the lower bounds of the dimensions of the invariant sets associated with the systems $(3,\{0,1 / n, 1\})$. First, if $n=2 \bmod 3$, the dimension of the sets is 1 , by Theorem 1.1. In addition if $n=3^{k}$ one can show (as in the calculation for $n=3$ done in Section 2) that the dimension of the set is approximately 0.876 ; this is also mentioned in [Kenyon 1997]. Therefore the displayed sequence cannot converge. Indeed, from its appearance, it might have many limit points.

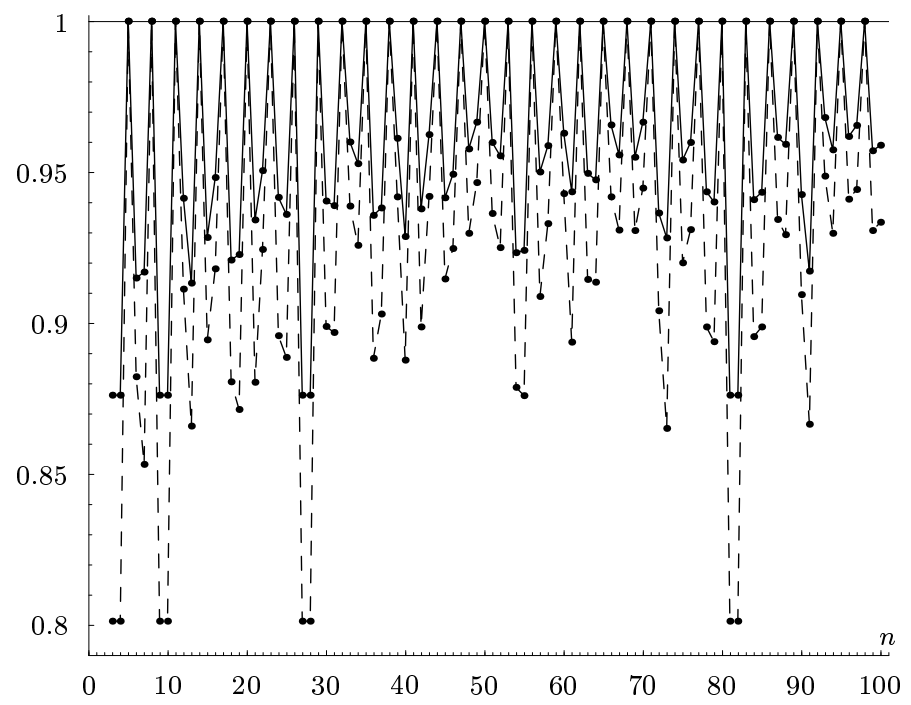

FIGURE 4. Dimension calculation when $t \rightarrow 0$. The system is $(3,\{0,1, n\})$; the upper (solid) curve gives the actual dimension, and the dashed curve gives the lower bound.

To make this more apparent, we calculated the lower bounds for the dimension for all $n \leq 1000$ for these systems and displayed them in Figure 5, which also shows the (exact) values for the dimension of the boundary of the invariant sets associated with the systems $(3,\{0,1 / n, 1\})$ when $n=2 \bmod 3$ (compare Theorem 2.6). They apparently converge (slowly) to 1; this was proved in [Kenyon et al. 1999]. From [Veerman and Jonker 1997], one can conclude that these boundaries as sets converge to $\Lambda_{t=0}$ in the Hausdorff metric.

There is a striking difference in the behavior of the dimension function depending on the limiting value of $t$ : when $t \rightarrow 1 / 2$ the dimension converge, and when $t \rightarrow 0$ they do not. From this we formulate a conjecture: 


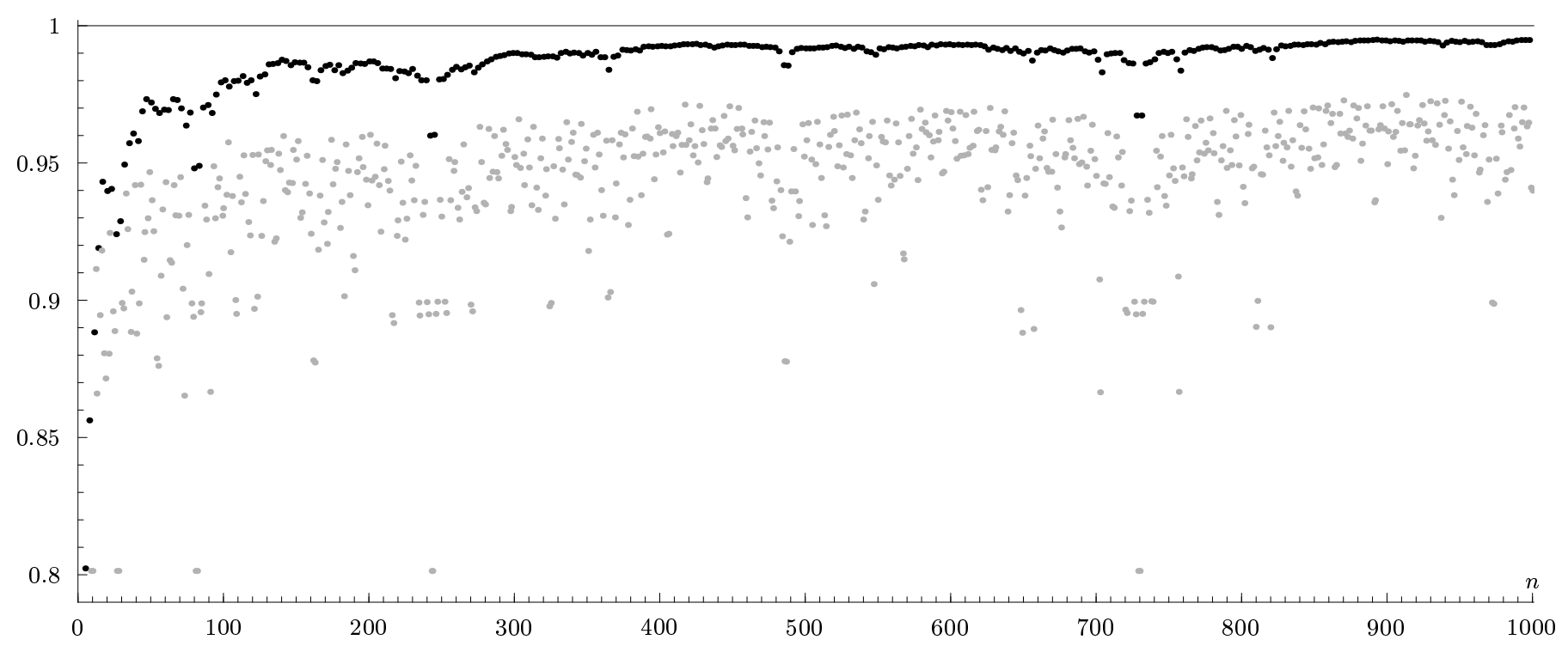

FIGURE 5. Dimension calculation when $t \rightarrow 0$ for large $n$. The system is $(3,\{0,1, n\})$; the black dots give the dimension of the boundary, and the gray dots give the lower bound for the dimension. Not shown are the gray $\operatorname{dots}$ at $(n, 1)$ for $n=2 \bmod 3$.

Conjecture 4.1. If $\lim _{t \rightarrow t_{0}} \operatorname{Hdim}(t)$ exists, $\operatorname{Hdim}(t)$ is continuous at $t_{0}$.

Based on this criterion we may now take the fact that the limit of the dimension as $t \rightarrow g$ appears to exist (see Figure 2), as evidence that $\operatorname{Hdim}\left(\Lambda_{t}\right)$ is continuous at the golden mean.

Because our main conjecture relies crucially upon this second conjecture, we felt it was necessary to provide additional evidence for it. Thus we tested the convergence of the bounds for the dimensions of the invariant of the system

$$
(3,\{0,(2+n) /(9+5 n), 1\}),
$$

where $t$ converges to $1 / 5$ and we expect the dimensions to converge to 1 (Figure 6 ), and those of the system

$$
(3,\{0,(3+2 n) /(8+5 n), 1\})
$$

where $t$ converges to $2 / 5$ and the dimensions are not expected to converge (Figure 7 ).

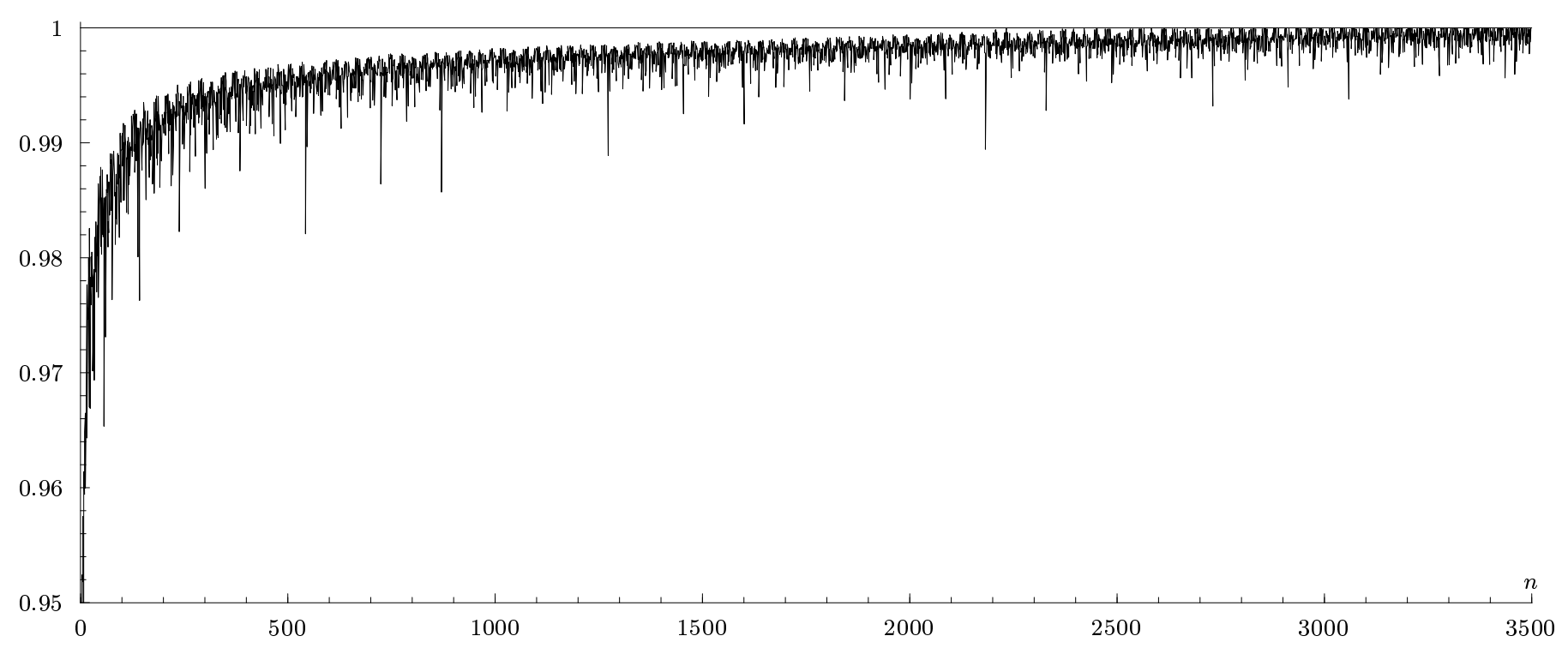

FIGURE 6. Dimension calculation when $t \rightarrow 1 / 5$. The system is $(3,\{0,2+n, 9+5 n\})$; only the lower bound of the dimensions is given. 


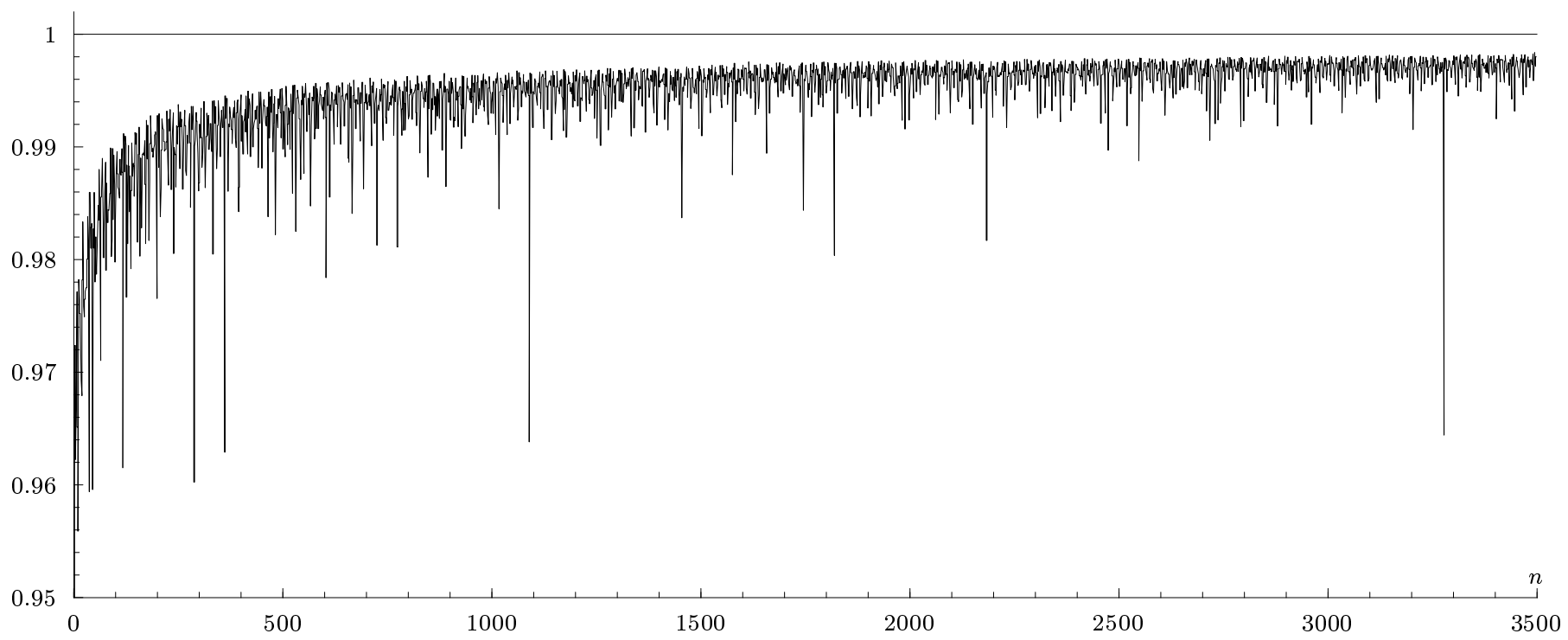

FIGURE 7. Dimension calculation when $t \rightarrow 2 / 5$. The system is $(3,\{0,3+2 n, 8+5 n\})$; only the lower bound of the dimensions is given. For clarity, those $n$ where the value is 1 are not shown.

The task of producing data for reasonable conclusions turned out to be computationally formidable. Only after a very careful, low level optimization of the $\mathrm{C}$ program (including consideration of register versus memory variable storage, inline function expansion, and substitution of algebraic operations by logical instructions), using two processors of the 64 bit UltraSparc HPC 3000 continuously for over forty days (60,000 minutes for each of the two processes, equivalent to three months CPU time), were we able to produce the data of Figures 6 and 7 .

These figures show only the lower bound, since the word algorithm that gives us the exact values of the dimension is not practicable except for very small values of $n$. However from the other figures it is apparent that the tendencies of the exact dimension are reflected in the behavior of the bound.

Finally, we also consider the invariant sets associated to the systems $\left(3,\left\{0, c_{n-1} / c_{n}, 1\right\}\right)$, where

$$
c_{n}=3 c_{n-1}+c_{n-2}, \quad c_{0}=1, \quad c_{1}=3,
$$

converging to the value $t=(\sqrt{13}-3) / 2 \approx 0.3027$. This should be similar to the golden mean case, except that now $p q=0 \bmod 3$ for all approximants. Numerically, this is a much harder problem than the case $t=g$; we were able to calculate only the lower bound of the dimension up to $n=7$. The results are shown in Figure 8, where it is seen that the dimension also seems to converge to 1 . The general behavior of successive approximants is similar to that in the golden mean case, except for the absence of points with $\operatorname{Hdim}(t)=1$ which satisfy the criterion given in Theorem 1.1(i).

In all rational cases that we have been able to check, we find that whenever $\operatorname{Hdim}\left(\Lambda_{p / q}\right)<1$ we also have that the lower bound of the dimension is strictly smaller than the (exact) dimension. This leads us to believe that in these cases the dimension of the support of the invariant measure is strictly smaller than the dimension of the set. That this should occur in one-dimensional systems was apparently not known, although it has been observed

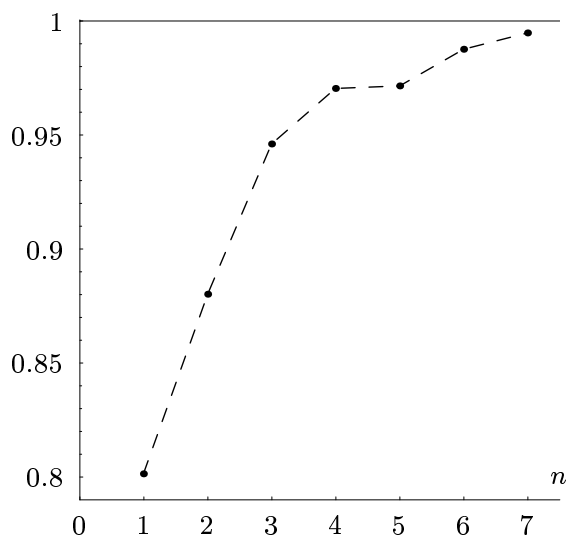

FIGURE 8. Dimension calculation when $t$ approaches $(\sqrt{13}-3) / 2$. The system is $\left(3,\left\{0, c_{n-1}, c_{n}\right\}\right)$, where the $c_{i}$ are defined in (4-2); the dots show the lower bound of the dimension. 
for two-dimensional iterated function systems [King 1995]. It is interesting to note that since the estimate of the dimension tends to 1 (in the third sequence) apparently the dimension of the measure in the irrational case equals the dimension of the set.

Conclusion. Our numerical work indicates that

$$
\operatorname{Hdim}\left(\Lambda_{g}\right)=\operatorname{Hdim}\left(\nu_{g}\right)=1
$$

where $g$ is the golden mean.

\section{ACKNOWLEDGEMENTS}

It is a pleasure to thank Leo Jonker, Sze-Man Ngai, Boris Solomyak, Greg Swiatek, and Yang Wang for their many helpful comments and references to the literature. We also thank the referees for several references to the literature.

\section{REFERENCES}

[Hacon et al. 1994] D. Hacon, N. C. Saldanha, and J. J. P. Veerman, "Remarks on self-affine tilings", Experiment. Math. 3:4 (1994), 317-327.

[Hutchinson 1981] J. E. Hutchinson, "Fractals and selfsimilarity", Indiana Univ. Math. J. 30:5 (1981), 713747 .

[Keane and Smorodinski 1997] M. Keane and M. Smorodinski, " $\beta$-expansions", preprint, 1997.

[Kenyon 1997] R. Kenyon, "Projecting the one-dimensional Sierpinski gasket", Israel J. Math. 97 (1997), 221-238.

[Kenyon et al. 1999] R. Kenyon, J. Li, R. S. Strichartz, and Y. Wang, "Geometry of self-affine tiles, II", Indiana Univ. Math. J. 48:1 (1999), 25-42. See http:// www.math.gatech.edu/ wang/reprints.html.
[King 1995] J. F. King, "The singularity spectrum for general Sierpiński carpets", Adv. Math. 116:1 (1995), $1-11$.

[Lagarias and Wang 1996] J. C. Lagarias and Y. Wang, "Integral self-affine tiles in $\mathbb{R}^{n}$, I: Standard and nonstandard digit sets", J. London Math. Soc. (2) 54:1 (1996), 161-179.

[Lau 1993] K.-S. Lau, "Dimension of a family of singular Bernoulli convolutions", J. Funct. Anal. 116:2 (1993), $335-358$.

[Oxtoby 1980] J. C. Oxtoby, Measure and category: A survey of the analogies between topological and measure spaces, 2nd ed., Graduate Texts in Math. 2, Springer, New York, 1980.

[Pollicott and Simon 1995] M. Pollicott and K. Simon, "The Hausdorff dimension of $\lambda$-expansions with deleted digits", Trans. Amer. Math. Soc. 347:3 (1995), 967-983.

[Rao and Wen 1998] H. Rao and Z.-Y. Wen, "A class of self-similar fractals with overlap structure", Adv. in Appl. Math. 20:1 (1998), 50-72.

[Solomyak 1995] B. Solomyak, "On the random series $\sum \pm \lambda^{n}$ (an Erdős problem)", Ann. of Math. (2) 142:3 (1995), 611-625.

[Veerman 1995] J. J. P. Veerman, "Intersecting selfsimilar Cantor sets", Bol. Soc. Brasil. Mat. (N.S.) 26:2 (1995), 167-181.

[Veerman 1996] J. J. P. Veerman, "Two-dimensional generalizations of Haar bases", pp. 220-235 in International Conference on Dynamical Systems (Montevideo, 1995), edited by F. Ledrappier, Pitman Res. Notes in Math. 362, Longman, Harlow, 1996.

[Veerman 1998] J. J. P. Veerman, "Hausdorff dimension of boundaries of self-affine tiles in $\mathbb{R}^{N}$, Bol. Soc. Mat. Mexicana (3) 4:2 (1998), 159-182.

[Veerman and Jonker 1997] J. J. P. Veerman and L. B. Jonker, "Rigidity properties of locally scaling fractals", preprint, IMS Stony Brook, 1997.

J. J. P. Veerman, Department of Mathematics, Pennsylvania State University, University Park, PA 16802, United States (veerman@math.psu.edu)

B. D. Stošić, Physics Department, Universidade Federal de Pernambuco, Recife, PE 50670-901, Brazil (e-mail:borko@lftc.ufpe.br)

Received December 7, 1998; accepted in revised form December 29, 1999 